\title{
Die Absorption von Stickstoff durch Calcium und seine Legierungen.
}

\author{
Von Otro Rufe und Hehimuth Hartmann.
}

Mit 1 Figur im Text.

Für die Verwendbarkeit des Calciums zur Absorption von Stickstoff $^{1}$ ) ist vor allem die Geschwindigkeit maßgebend, mit welcher der Stickstoff gebunden wird.

Nach den bisherigen Annahmen wird diese Geschwindigkeit einesteils durch die Temperatur, andernteils dureh die Struktur des Metalles bestimmt. Bezüglich der Temperatur meint Brandt festgestellt zu haben ${ }^{2}$ ), daß die Absorptionsgeschwindigkeit oberhalb der Schmelztemperatur $\left(800^{\circ}\right)$ ganz außerordentlich groß ist; unterhalb der Schmelztemperatur bis $660^{\circ}$ fast Null wird, dann wieder bis gegen $440^{\circ}$ steigt und schließlich endgültig fällt, - und bezüglich der Struktur, daß ein grobkristallines Metall rascher reagiert als ein feinkristallines.

Im Gegensatz dazu haben wir beobachtet, daß reines, im luftleeren Raum umgeschmolzenes Calcium, unabhängig davon, wie rasch es erstarrt ist bzw. welche Korngröße sein Gefüge besitzt, unterhalb seiner Schmelztemperatur praktisch indifferent gegen Stickstoff ist.

Die Ermittlung der Ursachen für diese Verschiedenheit der Beobachtungen und die Gewinnung einer möglichst sicher wirkenden Calciumlegierung war das Ziel dieser Arbeit.

Die Verschiedenheit der Beobachtungen konnte nur durch irgendeine Verunreinigung des von BRANDT verwendeten Metalls veranlaßt sein, sei es durch irgendein Fremdmetall, sei es auch durch Calciumnitrid. Brandt selbst hat beobachtet, daß die Reaktion

1) Hemper, Gasanalyt. Methode. Soddy, C. B. 1909, I, 235.

2) BRANDT, Dissertation, Leipzig 1915, „Über das metallische Calcium und seine Anwendung in der Gasanalyse"; sowie SteverT \& BrandT, Z. f. angew. Chem. 29 (1916) 402. 
dès Stickstoffs mit Calcium lebhafter wird, wenn die Oberfläche des Metalles erst einmal mit Nitrid bedeckt ist.

Unsere Versuche haben gezeigt, daß sowohl die Gegenwart von Calciumnitrid als auch diejenige gewisser Fremdmetalle unter bestimmten Bedingungen eine Beschleunigung der Stickstoffabsorption herbeiführt, und daß diese Beschleunigung durch die Herstellung entsprechender Calciumlegierungen in leicht reproduzierbarer Weise sjcher gestellt werden kann. Damit wird die bisherige Unsicherheit des Erfolgs aufgehoben. Man kann Legierungen herstellen, die in wenigen Minuten schon bei $<320^{\circ}$ das Argon aus atmosphärischem Stickstoff abzutrennen gestatten. Die niedrige Temperatur, bei der dieso Legierungen noch Stickstoff absorbieren, ermöglicht auch eine weitergehende Reinigung der Edelgase von Stickstoff, als dies bisher der Fall gewesen ist; denn das Optimum des erzielbaren Reinheitsgrades ist bestimmt durch den Dissoziationsdruck des Calciumnitrids, welcher mit der Temperatur natürlich sinkt.

\section{Die Herstellung der Calciumlogierungen.}

Ein Verfahren, Calciumlegierungen mit kloinem und bestimmtem Gohalt an Fremdstoffen in einwandsfreier Weise herzustellen, ist noch nicht bekannt. Die große Verwandtschaft des Metalls zu Sauerstoff, Stickstoff und Kohlensäure rnacht den Ausschluß der Gase nötig.

Man hat versucht den Zutritt der Gase dadurch zu beschränken, daß man über dem einzuschmelzenden Metall eine Decke von Calciumoxyd feststampfte ${ }^{1}$ ), oder aber dadurch, daß man das Metall unter einer Decke von Kalium-Natriumchlorid schmolz. Im ersten Fall verbrennt ein Teil des Metalls, der Rest wird nitridhaltig; im "wweiten bewirkt die größere Dichte des geschmolzenen Alkali(hlorids, daß das flüssig werdende Calcium teils durch die Salzdecke an die Oberfläche steigt und daselbst zu Oxyd verbrennt, teils aus ilem flüssigen Alkalichlorid Alkalimetall aufnimmt und zerstäubt bzw. in viele kleine Kügelchen zerteilt wird. Die in letzterem Fall gebildete Calcium-Alkalimetallegierung fängt an der Luft schon bei starkem Stoß oder Schlag, mitunter auch schon von selbst Feuer.

Das Schmelzen von Calcium macht auch im luftleeren Raum einige Schwierigkeiten; denn das Metall verdampft bereits unterhalb seincr Schmelztemperatur $\left(800^{\circ}\right)$ in erheblichem Umfang, z. B.

\footnotetext{
1) Dissertation BRandT, s. o.
} 
ist es KNOоHE') gelungen, es im hochevakuierten Rohr schon bei $400^{\circ} \mathrm{zu}$ sublimieren.

So bleibt nichts übrig, als das Motall in einem gasdicht geschlossenen Gefäß oder in einer Atmosphäre von reinem Edelgas zu legieren.

Das erste Verfahren ist das allgemeiner verwendbare; wir haben es in zwei Ausführungsformen benutzt.

a) Man verwendet sorgsam gereinigte, kurz ausgeglühte eiserne $\theta^{2}$ ) Tiegel oder Hülsen, entweder geschweißt aus Blech oder gedreht aus Rundstäben, stampft in diese die Metallmischung in Form reiner, grober Späne fest ein, drückt den oberen Rand flach zusammen, feilt ihn sauber und verschweibt ihn. (Die Wirkung des in den Tiegeln bei solchem Verfahren verbleibenden Juftrestes ist praktisch bedeutungslos.) Die geschlossenen Tiegel werden von Wasserstoff umspült in einem Röhrenofen $1 / 2$ Stunde lang auf $1000^{\circ}$ erhitzt. ${ }^{3}$ )

b) Schwach konische Eisentiegel werden mit dem Metall vollgestampft, mit einem Drahtnetz verschlossen und dann umgedreht, mit der Öffnung nach unten in einen weiteren Tiegel eingesetzt, in welchem sich eine geschmolzene Mischung von 100 Teilen Calciumchlorid und 16,5 Teilen Calciumfluorid befindet. Beim Erhitzen verschließt das geschmolzene Salzgemisch die untere Öffnung des Eisentiegels und hält die Luft von dessen Inhalt fern. ${ }^{3}$ )

Die Reguli waren bis auf den arsenhaltigen glatt geschmolzen, hatten aber oft einen sehr grob kristallinen Bruch und darum geringen Zusammenhalt. Damit sie möglichst unverletzt aus dem Tiegel genommen werden konnten, wurde dicser der Länge nach aufgesägt, und die Tiegelwand vom Regulus abgewickelt. Es ging

1) C. B. 1909, I, 52 .

2) Eisen löst sich in Calcium bis zu $1000^{\circ}$ nur wenig. Silikat- u. Magnesia. tiegel werden von Caloium angegriffen; Calcium nimmt Silicium, Aluninium, Magnesium aus deren Wandung auf.

3) Wir haben auch Tiegel aus gebranntem Kalk hergestellt. Kalk saugt geschmolzenes Calcium begierig auf; es bildet sich eine grate, beim Anfeilen schwach glänzende Masse, in welcher Kalk und Calcium ineinander gelöst sind Ws bleibt noch zu entscheiden, ob sich dabei ein Suboxyd bildet oder ob eine Lösung von Calcium in Calciumoxyd vorliegt.

In Dampfform wirkt Calcium auf Calciunoxyd nicht ein. Wenu also ein Suboxyd bestehen sollte, so wäre dessen Partialdruck für C'alciunn bei böheren Temperaturen größer als derjenige des reinen Calciums. Vielleicht könnte mau durch eine Extraktion des calciumoxydhaltigen Calciums mit fluissigem Ammoniak die Frage entscheiden.

4) Zum Patent angemeldet. 


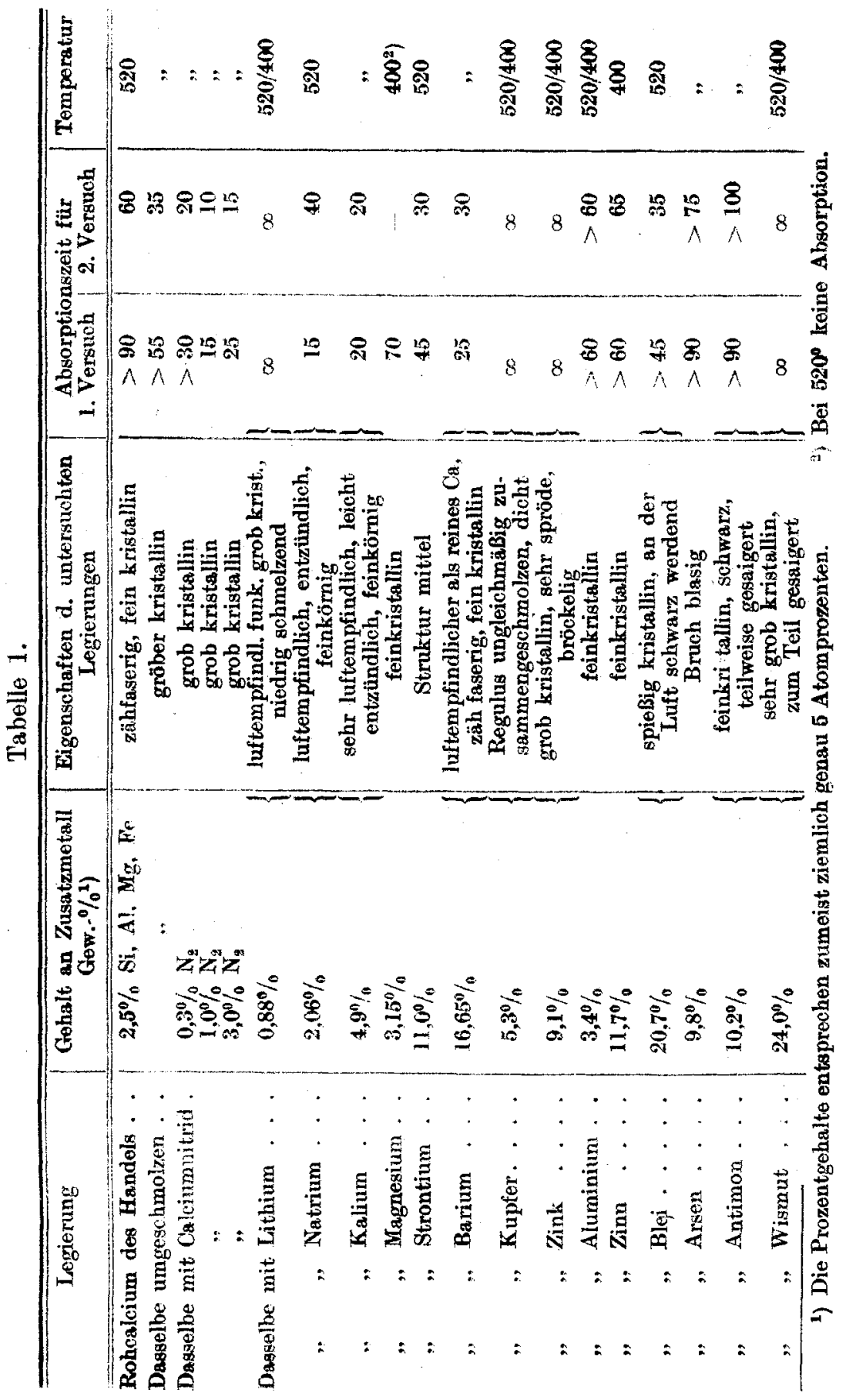


dies leicht, weil die Reguli außen mit einer dünnen Haut von Calciumoxyd überzogen waren, welche aus dem beim Ausglühen der Tiegel vor dem Füllen entstandenen Fisenoxyd nach der Gleichung

$$
\mathrm{Fe}_{2} \mathrm{O}_{3}+3 \mathrm{Ca}=3 \mathrm{CaO}+2 \mathrm{Fe}
$$

entstanden war. Wenn ein Tiegel nicht dicht gehalten hatte, so war der Inhalt nicht sauber zusammengeschmolzen, hing meist am oberen Ende und war von zahlreichen großen Blasen durchsetzt. Zur Vorbereitung für die Absorptionsversuche und um die Eigenschaften kennen zu lernen, wurden die Reguli auf der Drehbank auf kleine Zylinder von $8 \mathrm{~mm}$ Durchm. und $10 \mathrm{~mm}$ Länge abgedreht; sie erhielten damit alle dieselbe Oberfläche. Die dabei abfallenden sauberen Späne wurden gesammelt und zur quantitativen Bestimmung des Gehaltes an Zusatzmetall (vgl. Tabelle 1) verwendet.

Besonders bemerkenswert erscheinen in der Tabelle vor allem die Legierungen der Alkalimetalle. Sie sind ziemlich luftemptindlich, vom Lithium zum Kalium in steigenden Maße. Während bei der Lithiumlegierung nur kleine, besonders heiße Späne funken, entzünden sich bei der Kaliumlegierung auch schon die großen Späne, welche beim Abdrehen entstehen.

Einen merkwürdig blasigen Bruch zeigt die Arsenlegierung, einen abnorm grobkristallinen diejenige des Zinks und die des Wismuts. Saigerung wurde bei den Legierungen des Antimons und Wismuts, nicht aber des Bleis beobachtet.

In den Legierungen mit Calciumnitrid ist das Nitrid entweder fest gelöst oder aber mit mehr Calcium in noch unbekannter Form verbunden. Eine metallographische Untersuchung der CalciumStickstofflegierungen wird die Frage voraussichtlich zu entscheiden gestatten.

\section{Die Stickstoffabsorption.}

Die Absorptionsversuche wurden in dem von Brandt-Sieverts ${ }^{1}$ ) angegebenen Apparat zur Analyse von Edelgasen ausgeführt. Zur Heizung wurde aber statt eines einfachen Brenners ein kleiner Heraeusofen verwendet, der die genaue Messung der Temperatur gestattete.

Das Volumen des Absorptionsraumes wurde durch einen in das dbsorptionsrohr eingesetzten gläsernen Füllkörper möglichst ver. ringert und durch Druckmessungen mittels eines Hilfsvolumens ermittelt.

1) Z. angeel. Chem. 29 (1016), 402. 


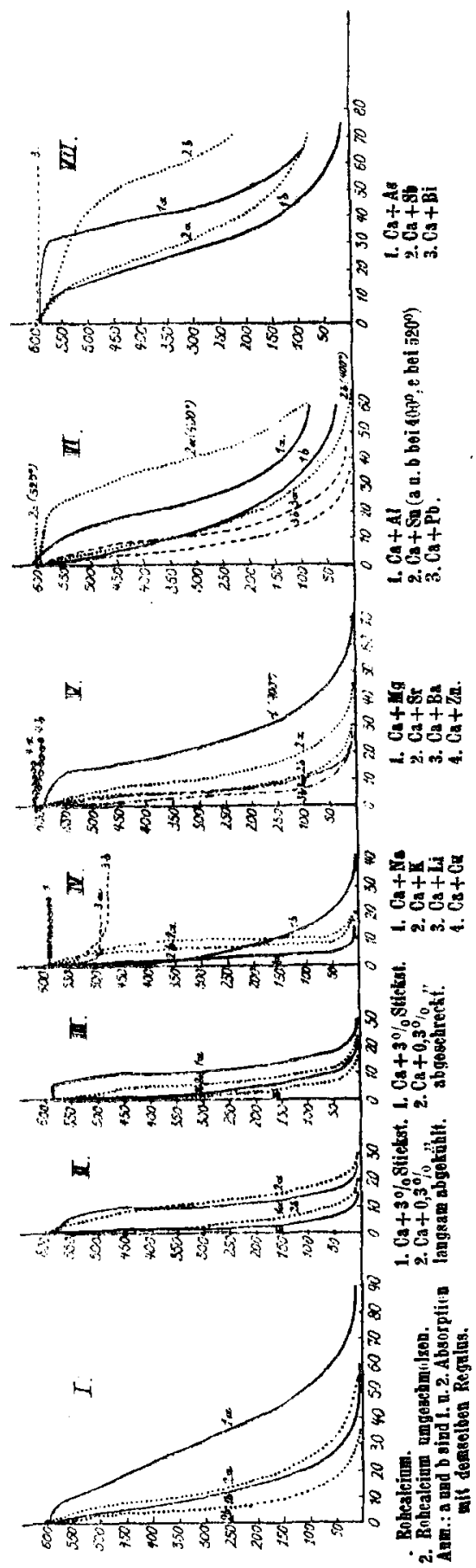

Bei jedem Versuch wurde die Apparatur zunächst vollkommen evakuiert, dann der Ofen angeheizt. In der Mebrzahl der Fälle war die Versuchstemperatur $520^{\circ}$, weil sich diese T'emperatur in der Apparatur besonders leicht konstant hailen lieB; nur soweit ein besonderes Intcresse vorlag, wurde auch bei anderen Temperatures gearbeitet. Wonn I'omperaturkonstanz eingetroton wir, wurde eine bestimmte Menge atmosphärischen Stickstoffs eingelassen. Der Anfangsdruck wurde: rasch abgelesen, die Ablesungen wurden alle 5 Minuten wiederholt. Dic abgelesenen Drucke wurden als Ordinaten, die zugehörigen Zeiten als Abszissen zur Zeichnung von Kurven verwendet.

Mit derselben Legierung wurden stets zwei, oft auch drei Versucho hintereinander ausgeführt.

Die Mehrzahl der Absorptionskurven sind in der nebenstehenden Figur wiedergegeben.

Die einer solchen Kurve, z. B. derjenigen mit 5 Atomprozenten $\mathrm{Na}$, zugrunde liegenden Beobachtungen sind in l'abelle 2 enthalten.

Der Verlauf der Kurven ist verschieden. Bei den meisten steigt dio Geschwindigkeit der Alssorption zunëchst langsamer an, behält dann einige Zeit ilhren Wort und fällt schließlich wieder mit sinkendem Sticlistoffdruck. Ein solcher Verlauf ist kennzeichnend für die autokatalytische Reaktionsbeschleunigung und am ausgesprochensten bei der 
ersten Absorption des Rohcalciums zu beobachten. Der Katalysator kann in diesem Fall nur ein an Calcium gesättigtes Nitrid sein. Wenn größere Mengen des Nitrids von Anfang an vorhanden sind, wie jm Rohcalcium nach der ersten Absorption oder in dem mit Nitrid legierten Calcium, ist der anfïngliche Anstieg der an sich schon erheblichen Geschwindigkeit geringer oder nicht mehr zu beobachten. Da ein solcher Anstieg auch bei den Legierungen mit Natrium, Kalium, Strontium und Barium entweder fehlt oder nur andeutungs. weise zu finden ist, und die Absorptionsgeschwindigkeiten auch bei diesen trotzdem größere Werte erreichen, so ist zu folgern, daß diese Metalle, also die dem Calcium gegenïber positiveren, ähnlich beschleunigend wirken, wio das Nitrid.

Tabelle 2.

Barometerstand $757 \mathrm{~mm}$. Temperatur $520^{\circ}$.

\begin{tabular}{|c|c|c|c|c|c|c|c|}
\hline \multirow[b]{2}{*}{$\begin{array}{c}t \\
\min \end{array}$} & \multicolumn{3}{|c|}{ Erste Absorption, } & \multicolumn{4}{|c|}{ Zweite Absorption. } \\
\hline & $p_{1}$ & $p_{2}$ & $p_{u}$ & $\begin{array}{c}t \\
\min \end{array}$ & $p_{1}$ & $p_{\mathbf{g}}$ & $p_{0}$ \\
\hline 0 & 540 & 376 & 593 & 0 & 540 & 376 & 593 \\
\hline 5 & 787 & 87 & 57 & 5 & 693 & 198 & 262 \\
\hline 10 & 810 & 62 & 9 & 10 & 737 & 147 & 167 \\
\hline 15 & 810 & 61 & 8 & 15 & 770 & 109 & 96 \\
\hline & & & & 20 & 789 & 86 & 64 \\
\hline & & & & 25 & 801 & 72 & 28 \\
\hline & & & & 30 & 807 & 66 & 16 \\
\hline & & & & 35 & 810 & 62 & 9 \\
\hline & & & & 40 & 810 & 61 & 8 \\
\hline
\end{tabular}

Die anderen Metalle lassen die Absorptionsgeschwindigkeit des umgeschmolzenen Rohcalciums entweder praktisch unverändert (Al, $\mathrm{Mg}, \mathrm{Pb}, \mathrm{Sn}$ ) oder verlangsamen sie (As, Sb) oder heben sie ganz auf (Bi, $\mathrm{Cu}, \mathrm{Zn}$ ).

Fin Einfluß der Größe der Kristallite der Legierungen hat sich nicht mit Sicherheit feststellen lassen. Zwar absorbiert das einmal umgeschmolzene, gröberkiristalline Rohcalcium rascher als das frische. Die Beschleunigung dürfte aber, da beim Umschmelzen der Stickstoffgehalt des Metalls um ein weniges erhöht wird, weniger durch die Veränderung der Struktur als durch die Vermehrung des Nitridgehaltes verunlaßt worden sein. Bleibt der Nitridgehalt unverändert, so absorbiert das Metall im abgeschreckten kleinkristallinen Zustand eher rascher als im langsam gekühlten, grobkristallinen. Auch zeigen gerade einige der schlechter oder gar nicht absorbierenden Legierungen, wie z. B. diejenigen des Antimons, Zinks und Wismuts ein besonders grobkristallines Gefüge. 
Die größere Absorptionsgeschwindigkeit einmal umgeschmolzenen und langsam abgekühlten Metalls, welche Brandt (s. v.) nicht zureichend auf dessen gröber kristalline Struktur zurüokgeführt hat, findet damit eine ausreichende Erklärung. BrandT hat sein Metall unter einer gestampften Decke von Calciumoxyd in einem Eisenrohr an der Luft geschmolzen und dann langsam erkalten lassen; dabei mußte sich eine gewisse Menge Nitrid bilden und zwar um so mehr, je langsamer das Metall erkaltete, und von je höherer Temperatur aus die Abkühlung vor sich ging, - im Einklang mit Brandts Beobachtungen.

In einigen Fällen, so z. B. beim Zink, Arsen, Antimon bildet das Zusatzmetall Verbindungen ${ }^{1}$ ) mit dem Calcium. Die 5 Atomprozente Zusatzmetall dürften in keinem Fall ausreichen, alle Calciumatome zu binden, und auch kaum dazu, dem Stickstoff eine Einwirkungsgrenze zu setzen ${ }^{2}$ ), selbst wenn eine besonders hohe Koordinationszahl angenommen wird (z. B. etwa $\mathrm{ZnCa}_{10}$ ).

Die verschiedenen Kurven lassen sich unter diesen Umständen nicht durch eine gemeinschaftlich mathematische Beziehung darstellen. Wegen der katalytisehen Beschlounigung durch das Reaktionsprodukt (Nitrid) und der dadurch bedingten Inkonstanz der Oberfläche versagen die bekannten Gleichungen für die Reaktionsgeschwindigkeit in heterogenen Systemen; die Nitridbildung setzt sich an verschiedenen Stellen der Oberfläche mit recht versehiedener Geschwindigkeit nach der Tiefe zu fort. Aber alle Kurven laufen asymptotisch einem Grenzwert des Druckes zu, welcher durch den Rohargongehalt des Luftstickstoffs $\mathrm{zu}$ rund $6,4 \%$ bestimmt ist. Die Zeit, innerhalb welcher dieser Grenzwert erreicht wird, kann darum als Maß für die Wirkung des Zusatzes dienen. Sie ist in Tabelle 1 in den beiden letzten Vertikalreihen für jede einzelne Legierung, und zwar sowohl für die erste, als auch die daran sich anschliekende zweite Absorption mitgeteilt worden.

Der Einfluß der Temperatur auf die Absorptionsgeschwindigkeit ist bei Calcium mit $1,0 \%$ Stickstoff etwas eingehender verfolgt worden. Das Ergebnis der Versuche ist in Tabelle 8 zusammengestellt.

Also selbst bei $320^{\circ}$ und darunter lassen sich bei ausreichendem Gehalt an Nitrid ganz erhebliche Absorptionsgeschwindigkeiten erzielen.

1) Doxgki, 2. anorg. Chem. 67 (1908), 185.

2) Tumani, Z. anorg. u. allg. Chem. 107 (1919), 107. 
Tabelle 3.

\begin{tabular}{|c|c|c|c|c|}
\hline Temp. & $\begin{array}{l}\text { Dauer d } \\
\text { 1. Versuch }\end{array}$ & $\begin{array}{l}\text { Absorption } \\
\text { 2. Versuch }\end{array}$ & $\begin{array}{l}\text { Minuten } \\
\text { 3. Versuch }\end{array}$ & Bemerkungen \\
\hline 320 & 15 & 5 & 7 & $\begin{array}{l}\text { während der 2. u. 3. Ab- } \\
\text { sorption Ansteigen der } \\
\text { Temp. auf } 330 \text { bzw. } 340^{\circ}\end{array}$ \\
\hline 400 & 10 & 5 & 5 & $\begin{array}{l}\text { während der 2. Absorp- } \\
\text { tion Ansteigung der Tem- } \\
\text { peratur auf } 410^{\circ}\end{array}$ \\
\hline $\begin{array}{l}500 \\
620\end{array}$ & $\begin{array}{l}15 \\
25\end{array}$ & $\begin{array}{l}10 \\
25\end{array}$ & 8 & por \\
\hline 740 & 60 & 30 & - & $\begin{array}{l}\text { nach I glatte, gesintorte } \\
\text { Decke von Nitrid }\end{array}$ \\
\hline 745 & 50 & 20 & - & 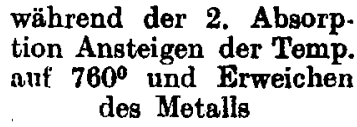 \\
\hline
\end{tabular}

Die Gründe für die verschiedene Geschwindigkeit der Stickstoffabsorption liegen also teils auf physikalischem Gebiet, in der Beschaffenheit und Entwicklung der Oberfläche, sowie den Volumverhältnissen beim Umsatz, teils auf chemischem.

Damit die Absorption bis zum völligen Verbrauch des Metalls fortschreiten kann, muß dessen Oberfläche dauernd durchlässig für Stickstoff bleiben; es darf als Folge der Stickstoffaufnahme keine Verdichtung der Oberfläche eintreten.

Für reines Calcium und für Temperaturen, welche wesentlich unterhalb seiner Schmelztemperatur liegen, geschieht dies auch nicht. Es tritt nicht bloß keine Volumvermehrung, sondern eine Volumverminderung ein; denn das Atomvolum des Calciummetalls ist größer als dasjenige des daraus hervorgehenden Nitrids:

$\begin{array}{ccccc}\text { Dichte des Calciums für } 1,59 ; & \text { Atomvolum } & 25,214 \\ ,, \quad \text { "Stickstoffs für } 1,0265 ; \quad, & 18,648 \\ , \quad \text { ". Nitrids }{ }^{1} \text { ) } \quad 2,7006 ; & \text { Mol }, & 54,80 \\ & \text { Atomvolum von } 3 \mathrm{Ca} & =75,64 & \\ \text { Molekularvolum von } \mathrm{Ca}_{3} \mathrm{~N}_{2}=54,80 & \end{array}$

Die Folge dieser eigentümlichen Volumverhältnisse ist, dal die Nitridschicht für den Stickstoff durchlässig bleibt.

Erlaubt höhere Temperatur einen beschränkten Platzwechsel der Calciumatome und Nitridmoleküle, so schrumpft die Nitridschicht zusammen; die Oberfläche wird dichter und die Absorption langsamer. Dadurch ąber, daß sich die Absorption nach der Tiefe zu ungleichmäBig fortsetzt, treten in der Oberfläshe Spannungen auf;

1) Bei $700^{\circ}$ dargestellt. 
die Oberfläche wird bei Temperaturwechsel leicht rissig und läßt das Gas wieder in beschränlitem Umfang durchtreten.

Bei den Legierungen treten Komplikationen auf. Das Zusatzmetall kann den Platzwechsel der Atome begünstigen, indem es die Schmelztemperatur erniedrigt, aber auch im entgegengesetzten Sinne wirken. Chemisch verhält es sich bald ähnlich dem Calcium und kann ihm parallel Nitrid bilden ( $\mathrm{Al}, \mathrm{Mg}, \mathrm{Li}$ ), bald ist es dem Stickstoff gegenüber praktisch indifferent und wird die Reaktion des Calciums mit Stickstoff dann nicht sehr stören $(\mathrm{Pb}, \mathrm{Sn})$, bald geht es auch mit dem Calcium eine Verbindung ein und kann dann praktisch indifferent $(\mathrm{Pb}, \mathrm{Sn})$ oder verlangsamend wirken $(\mathrm{As}, \mathrm{Sb}, \mathrm{Bi}$, $\mathrm{Zn}, \mathrm{Cu})$. Nur in wenigen Fällen wirkt der Zusatz ausgesprochen begünstigend ( $\mathrm{Na}, \mathrm{K}, \mathrm{Ba}, \mathrm{Sr}$ ).

Welche Umstände im einzelnen Fall vorliegen, ist auf Grund der vorliegenden Versuche allein nur in wenigen Fällen entgültig zu entscheiden. Saigerungen haben wir beim Wismut, Antimon und Lithium beobachtet; sie dürften die Verzögerung und beim Lithium den Stillstand der Reaktion veranlassen. Auch die Verzögerung der zweiten Absorption bei der Natriumlegierung dürfte in einer solchen eine befriedigende Erklärung finden, weil der Natriumgehalt ihres Kernes während der Absorption stieg. Aber wichtiger als diese mehr äußerlichen Beobachtungen erscheint uns eine andere - nämlich die, daß es die dem Calcium gegenüber positiveren Elemente sind, welche die Reaktion beschleunigen, und die negativeren; welche sie verlangsamen. Die Zugabe von positiveren Metallen hat eine Lockerung, diejenige von negativeren eine festere Bindung der Valenzelektronen des Calciums zur Folge. Von welcher Bedeutung eine solche Änderung der Festigkeit der Elektronenbindung für das Eintreten oder Ausbleiben einer Reaktion wird, dürfte besser erst dann erörtert werden, wenn noch mehr Beobachtungsmaterial. dazu vorliegt.

\section{Zusammenfassung.}

Calcium und calciumreiche Legierungen lassen sich in einem gegen die Luft abgeschlossenen Eisentiegel schmelzen. Den Abschluß erreicht man in befriedigender Weise, wern man die Tiegelöffnung entweder autogen verschweißt oder durch eine Calciumchloridfluoridschmelze schließt, in welche der Tiegel mit der Öffnung: nach unten eingesetzt wird. 
Die Reaktionsgeschwindigkeit solcher Legierungen mit Stickstoff wird hauptsächlich von den Volumverhältnissen, der Temperatur und dem Potential des Zusatzmetalles, sowie von dem Gehalt der Legierung an Calciumnitrid beeinfluBt. Die starke Volumverminderung des Calciums bei der Bildung des Nitrids erhält die Außenschicht des Metalles porös, solange sie keine Sinterung oder Saigerung verdichtet bzw. eine zu hohe Temperatur ein teilweises Schmelzen veranlaBt. Trotzdem erscheint völlig reines Calcium gegen Stickstoff ganz oder nahezu passiv. Nur diejenigen Metalle beschleunigen die Stickstoffbindung, welche positiver als das Calcium sind, wäbrend die negativeren auf die Bindung entweder ohne Einfluß sind oder sie verlangsamen. Eine Sonderstellung hat das Calciumnitrid, dessen beschleunigende Wirkung sich bei allen Calciumlegierungen bemerkbar macht, wenn sie an der Luft umgeschmolzen worden sind.

Ein solcher Nitridgehalt ist auch die Ursache dafür, daß ein bei höherer Temperatur unter einer Decke von Calciumoxyd umgeschmolzenes und langsam erkaltetes Calcium Stickstoff besser absorbiert als frisches. Die damit verbundene Vergröberung der Struktur des Metalls, welche BrandT für wesentlich hält, ist der Vergrößerung des Nitridgehaltes gegenüber ziemlich bedeutungslos. Mit einer etwa 5\% nitridhaltigen Calciumlegierung läßt sich aus atmosphärischem Stickstoff schon bei $<320^{\circ}$ in wenigen Minuten reines Argon gewinnen.

Breslau, Anorganisch-chemisches Institut der Technischen Hochschule.

Bei der Redaktion eingegangen am 9. Januar 1922. 